\title{
Traumatismes thoraciques non chirurgicaux
}

\section{Non-surgical Blunt Chest Trauma}

\author{
C. Laplace $\cdot$ A. Harrois $\cdot$ S. Hamada $\cdot$ J. Duranteau
}

Reçu le 2 octobre 2017; accepté le 22 décembre 2017

(C) SRLF et Lavoisier SAS 2018

Résumé Les traumatismes fermés du thorax peuvent être à l'origine de lésions engageant le pronostic vital d'emblée ou de lésions dont l'évolution peut s'aggraver dans les heures qui suivent le traumatisme. L'évaluation correcte et exhaustive des lésions est donc cruciale et la surveillance des premières heures, indispensable, ce d'autant que le traumatisme aura été violent ou que le patient présente des facteurs de risque. L'échographie pleuropulmonaire lors du déchoquage, puis la tomodensitométrie thoracique avec injection de produit de contraste, dans le cadre d'un bilan « corps entier », sont les examens indispensables à une évaluation adaptée chez ces patients fréquemment victimes d'un polytraumatisme et présentant des lésions extrathoraciques associées. La prise en charge non chirurgicale est fondée sur une analgésie rapidement optimale, une prise en charge ventilatoire intensive associant : drainage pleural efficace, oxygénothérapie à haut débit, ventilation non invasive, ventilation protective si l'intubation s'avère nécessaire. Enfin, la réanimation d'une insuffisance circulatoire doit être raisonnée, guidée par un monitorage multimodal invasif, particulièrement pour le remplissage vasculaire et les transfusions. Dans quelques cas, un patient traumatisé du thorax initialement considéré comme non chirurgical pourra finalement nécessiter une chirurgie. Cela ne doit pas être négligé pour une prise en charge dans des structures adaptées et une discussion avec des équipes pluridisciplinaires entraînées (stenting aortique, fixation d'un volet costal, exploration d'un hémothorax résiduel).

Mots clés Traumatisme thoracique fermé $\cdot$ Contusion pulmonaire · Pneumothorax · Hémothorax ·

Polytraumatisme

C. Laplace $(\bowtie) \cdot$ A. Harrois $\cdot$ S. Hamada $\cdot$ J. Duranteau Département d'anesthésie-réanimation chirurgicale, réanimation chirurgicale,

hôpitaux universitaires Paris-Sud, hôpital Bicêtre,

78, rue du Général-Leclerc,

F-94275 Le Kremlin-Bicêtre Cedex, France

e-mail : christian.laplace@aphp.fr
Abstract Blunt chest injuries are frequent in trauma patients and often tend to pose significant diagnostic and therapeutic challenges to physicians. Chest trauma may not only be lifethreatening because of immediate respiratory and/or hemodynamic failures but also because of delayed worsening of respiratory status within the hours following trauma. In both situations, chest trauma requires appropriate therapeutic intervention to prevent trauma death. For this reason, accurate and complete appraisal of thoracic injuries is crucial. Thoracic ultrasonography and FAST must be conducted in short notice in the trauma setting to evaluate hemodynamically unstable blunt trauma patients since it assesses rapidly vital injuries. After achieving vital signs stabilization, totalbody CT scanning must be performed to detect all thoracic injuries and potential extra-thoracic injuries. Management of patients with blunt chest trauma focuses on interventions such as chest drainage, pulmonary toilet, effective physiotherapy, and early and adequate pain control. Ventilatory support aims at preventing intubation by using high-flow oxygen therapy, continuous positive airway pressure, or non-invasive positive pressure ventilation. When endotracheal intubation and mechanical ventilation are required, the guidelines of protective ventilation must be applied. Hemodynamic resuscitation is guided by multi-modal monitoring to prevent fluid overload in these patients at high risk for developing respiratory failure. In some cases, blunt thoracic trauma requires delayed surgery. Therefore, trained trauma team should be involved in the decision of surgical, endovascular or non-surgical care when pleural active bleeding, flail chest or aortic trauma are identified.

Keywords Blunt chest trauma $\cdot$ Thoracic injuries $\cdot$ Lung injury $\cdot$ Pleural effusion $\cdot$ Pneumothorax

\section{Introduction}

Les traumatismes thoraciques sont des pathologies fréquentes. La Traumabase dénombre 1184 patients avec un Abbreviated Injury Scale (AIS) supérieur ou égal à deux, pris en 
charge dans l'un des six centres de traumatologie lourde d'Île-de-France pour l'année 2016. Dans le cadre de la traumatologie lourde et le plus souvent dans ce cas dans un contexte de polytraumatisme sévère, un tiers des polytraumatisés a une atteinte thoracique, et $80 \%$ des traumatisés $\mathrm{du}$ thorax ont d'autres lésions susceptibles d'engager le pronostic vital [1]. Ils représentent aussi une part importante de la traumatologie « bénigne », quotidienne dans les services d'accueil des urgences. Les lésions peuvent être d'une extrême variété avec mise en jeu du pronostic vital et associées à une mortalité de $25 \%$, ce qui fait du traumatisme thoracique la deuxième cause de décès en traumatologie [2]. Une des difficultés de l'évaluation des traumatismes thoraciques vient du fait qu'il n'existe pas de parallélisme entre les lésions pariétales et les lésions intrathoraciques viscérales, lesquelles s'expriment parfois de manière retardée [3]. Il convient donc de ne considérer un traumatisme thoracique comme non chirurgical qu'à l'issue d'une évaluation correcte et d'un bilan exhaustif mené de manière adaptée à la recherche de lésions possiblement occultes à l'examen clinique. Beaucoup de lésions, parfois initialement non évidentes, peuvent indiquer une chirurgie cardiothoracique (fixation de volet costal, exploration d'un hémothorax massif, plaie pulmonaire, rupture de gros vaisseau, complication cardiaque...), viscérale (rupture de coupole diaphragmatique, lésion œsophagienne), orthopédique (traumatisme rachidien) ou un traitement par voie endovasculaire (lésion aortique). On exclura de cette revue les traumatismes pénétrants qui sont le plus souvent chirurgicaux et restent encore à l'heure actuelle minoritaires en France. Nous nous concentrerons sur les traumatismes fermés, leur bilan, leur prise en charge et sur l'évaluation permettant d'éliminer les lésions chirurgicales éventuelles.

\section{Évaluation de la gravité d'un traumatisme thoracique}

Les mécanismes lésionnels peuvent être multiples, à type de choc direct, de compression thoracique, de blast ou de décélération. Ils sont volontiers associés entre eux et à l'origine de lésions pariétales et/ou intrathoraciques diverses [4]. L'énergie cinétique au moment du choc, la déformabilité du thorax (plus importante chez l'enfant et le jeune adulte que chez le sujet âgé) ainsi que le mécanisme prépondérant du choc seront à l'origine de lésions à prédominance pariétale (fractures de côtes, volets costaux...) ou internes à type de traumatisme direct ou de lésions de cisaillement et d'étirement (contusion pulmonaire, rupture isthmique aortique, lésions trachéobronchiques...) [5]. Les associations lésionnelles thoraciques peuvent être multiples, mais la violence du traumatisme responsable doit faire envisager systématiquement l'éventualité d'associations lésionnelles extrathora- ciques qui doivent être explorées (traumatisme crânien, traumatisme rachidien, traumatisme abdominal). La gravité doit donc être évaluée en considérant avant tout ces patients comme des victimes de polytraumatisme et non pas a priori comme des traumatisés du thorax isolé [1].

L'évaluation initiale s'attachera donc à mettre en évidence des signes de gravité immédiate, l'existence d'une détresse respiratoire en premier lieu. Celle-ci est liée à une atteinte de la mécanique ventilatoire aboutissant à l'inefficacité de l'effecteur pulmonaire ou à l'apparition d'inégalités de ventilation/perfusion par atteinte parenchymateuse. Le deuxième critère de gravité majeure est l'apparition d'une détresse hémodynamique secondaire, la plupart du temps, à un état de choc hémorragique, parfois par état de choc obstructif (pneumothorax compressif) ou cardiogénique. Les recommandations formalisées d'experts conjointes de la Société française d'anesthésie et de réanimation et de la Société française de médecine d'urgence, parues en 2015, retiennent les critères de gravité suivants [6] : la constatation d'une détresse respiratoire clinique avec une FR supérieure à 25/ min et/ou une hypoxémie $(\mathrm{SpO} 2<90 \%$ sous air ambiant ou $<95 \%$ sous oxygénothérapie) et la constatation d'une détresse circulatoire (chute de PAS $>30 \%$ ou PAS $<$ $110 \mathrm{mmHg}$ ). Enfin, les experts recommandent d'utiliser le score MGAP (Mechanism, Glasgow Coma Scale, Age and Arterial Pressure) afin de détecter les patients traumatisés à risque élevé de mortalité intrahospitalière. Le score MGAP est un score composite associant le score de Glasgow, le niveau de pression artérielle systolique, le caractère fermé ou pénétrant du traumatisme ainsi que l'âge supérieur ou égal ou inférieur à 60 ans du patient. Le score varie de 3 à 29. Un score compris entre 3 et 17 est associé à une mortalité prédite hospitalière de près de $50 \%$ [7].

Lors de traumatisme semblant mineur, ou lors d'un traumatisme sévère en l'absence de signes de gravité immédiate, il faut considérer comme à risque d'aggravation secondaire les patients âgés de plus de 65 ans, qui présentent une pathologie pulmonaire ou cardiovasculaire chronique, un trouble de la coagulation congénital ou acquis (traitement anticoagulant ou antiagrégant), lorsque la cinétique de l'accident est décrite comme violente (selon les critères de Vittel décrits en 2002 [8]) et lorsque le bilan met en évidence plus de deux fractures de côtes [6,9]. En effet, un élément majeur de l'évaluation de la gravité des traumatisés du thorax est que celleci s'évalue non seulement initialement, mais aussi de manière dynamique en fonction de l'évolution au cours des premières heures. Ces traumatismes sont à fort potentiel d'aggravation dans les 72 premières heures. Les aggravations secondaires résultent de l'évolution des lésions initiales, du retentissement pulmonaire des phénomènes systémiques (ischémie - reperfusion, inflammation, sepsis...) ou des effets délétères d'interventions thérapeutiques (remplissage massif, polytransfusion, ventilation agressive...) [10]. 
On retrouve particulièrement cette aggravation secondaire lors d'atteintes pariétales avec des fractures multiples de côtes et/ou l'existence de volets costaux ou lors de la mise en évidence d'une contusion pulmonaire. En présence de fractures de côtes multiples, l'évolution sera marquée par la possible apparition d'une détresse respiratoire secondaire, d'autant plus que l'analgésie aura été inadéquate et/ou insuffisante, avec inefficacité de la toux, encombrement, et atélectasie. Des complications secondaires d'origine pleurale sont fréquentes, à type d'hémothorax retardé ou de pneumothorax secondaire par déplacement osseux et lacération du parenchyme pulmonaire. En présence d'une contusion pulmonaire, l'évolution revêt classiquement un caractère biphasique, avec une atteinte immédiate corrélée à l'amputation traumatique du parenchyme pulmonaire fonctionnel, puis secondairement à une augmentation de taille de la lésion initiale par des mécanismes inflammatoires. La libération conjuguée de cytokines inflammatoires, d'espèces radicalaires et d'enzymes altère l'endothélium et la barrière alvéolocapillaire favorisant l'apparition d'un œdème lésionnel. Le parenchyme pulmonaire s'altère, et les lésions se bilatéralisent [11-14].

Un index d'oxygénation initial $\left(\mathrm{PaO}_{2} / \mathrm{FiO}_{2}\right)$ inférieur à 300 et qui reste inférieur à 300 à 24 heures du traumatisme, de même que l'existence d'un volume de contusion représentant plus de $20 \%$ du volume pulmonaire évalué sur la tomodensitométrie, signe une contusion pulmonaire sévère, avec un risque élevé de survenue d'une détresse respiratoire et une incidence élevée de SDRA au cours de l'évolution [15].

Cette difficulté d'évaluation au cours des premières heures justifie les recommandations d'experts qui préconisent [6] :

- une orientation vers un centre spécialisé de traumatologie de tout patient présentant un signe de gravité immédiate à type de détresse respiratoire et/ou circulatoire [16,17] ;

- un avis spécialisé systématique pour tout patient ayant des facteurs de risque d'aggravation secondaire ;

- une surveillance d'au moins 24 heures pour tout patient présentant des facteurs de risque d'aggravation secondaire.

\section{Quel bilan lésionnel doit être réalisé ?}

Le bilan lésionnel d'un traumatisé thoracique doit prendre en considération sa stabilité hémodynamique et respiratoire. L'enchaînement des examens d'imagerie doit être raisonné pour apporter les informations pertinentes au bon moment de la prise en charge. On doit garder à l'esprit que chaque traumatisé du thorax est possiblement un polytraumatisé présentant des lésions extrathoraciques. La phase de déchocage doit être standardisée et réalisée avec des équipes entraînées selon une séquence précise [18]. Lorsque le patient est instable sur le plan hémodynamique ou respiratoire à l'arrivée à l'hôpital, l'examen urgent à réaliser est l'échographie pleuropulmonaire [19]. Réalisé en complément de la FAST échographie (focused assessement with sonography for traumas comprenant une échographie abdominale et péricardique) et de la radiographie thoracique au déchoquage, cet examen permet rapidement de déterminer l'origine de l'instabilité en même temps que la réalisation des premières mesures de réanimation symptomatiques [6]. Il permet, devant l'instabilité hémodynamique, de déterminer si elle est liée à l'existence d'un hémothorax [20] et en association à la FAST, de déterminer s'il existe un épanchement abdominal associé ou un hémopéricarde avec tamponnade péricardique. Cet examen a été démontré comme permettant de prendre des décisions thérapeutiques urgentes appropriées [21]. Lorsque la détresse respiratoire est présente, l'échographie pleuropulmonaire décèle rapidement l'existence d'un pneumothorax. Le diagnostic est rapide [22]. Les arguments échographiques évocateurs de la possibilité d'un pneumothorax sont l'absence d'artéfact en queue de comète et la perte du glissement pleural. Le seul critère échographique pathognomonique dont la spécificité est de $100 \%$ est la mise en évidence du point poumon à l'endroit de la transition poumon à la paroi/poumon décollé. L'échographie est aussi spécifique que la radiographie thoracique, mais plus sensible ( 86 vs $28 \%$ ) pour le diagnostic de pneumothorax, car elle décèle les pneumothorax de faible abondance, invisibles sur la radiographie pulmonaire [22]. La radiographie de thorax est l'examen classique réalisé de manière concomitante. Elle permet de visualiser des fractures de côtes, les atteintes parenchymateuses et confirme les atteintes visualisées par l'échographie. En association avec la radiographie du bassin, elle fait partie de l'imagerie d'urgence d'un polytraumatisé instable, avant la réalisation du bilan exhaustif scannographique qui sera effectué après la stabilisation hémodynamique et respiratoire. En effet, le bilan complet du patient doit faire appel au scanner corps entier systématique, avec injection de produit de contraste $[23,24]$. Il s'agit du seul examen qui dépistera toutes les lésions thoraciques et extrathoraciques. Il a été démontré que la réalisation du scanner systématique a permis d'améliorer la prise en charge avec une augmentation du nombre de lésions décelées et une baisse de la mortalité [25].

Lorsque le patient présente des critères de gravité, mais est stable à la prise en charge, la tomodensitométrie thoracique avec injection en tant qu'élément de la tomodensitométrie corps entier est recommandée [6]. La réalisation d'une eFAST (FAST échographie + échographie pleuropulmonaire) réalisée avant le scanner permet de déceler $100 \%$ des pneumothorax qui nécessitent un drainage [26].

En complément de la tomodensitométrie corps entier systématique, ou lorsque celle-ci ne peut être réalisée pour des raisons de chirurgie immédiate, on peut associer l'échocardiographie transœsophagienne (ETO) pour le bilan des 
lésions traumatiques aortiques $[27,28]$. La tomodensitométrie, particulièrement depuis l'avènement des scanners multidétecteurs, permet une excellente définition des lésions vasculaires. Elle met en évidence toutes les lésions qui peuvent nécessiter rapidement une chirurgie ou un traitement endovasculaire. L'ETO, pour sa part, met en évidence ces lésions décelées par le scanner, mais aussi des lésions vasculaires supplémentaires, non chirurgicales, mais qui nécessitent une surveillance régulière afin de déceler des complications ultérieures éventuelles (déchirures intimales aortiques minimes). On peut ainsi suggérer de réaliser un examen par ETO chez tous les patients traumatisés sévères, d'autant plus que le mécanisme a impliqué une décélération violente. Cet examen, s'il est réalisé, le sera comme bilan exhaustif complémentaire secondaire, " à froid " et après avoir éliminé tout risque de lésion traumatique œsophagienne.

\section{Analgésie, composante majeure de l'évolution des premiers jours}

Le contrôle de la douleur est une urgence lors d'un traumatisme thoracique. Elle est l'un des éléments qui doivent être contrôlés sans délai. Une analgésie parfaite doit être assurée, aussi bien au repos qu'à la toux et à la mobilisation, afin de ne pas favoriser une dégradation de la fonction ventilatoire du patient. En effet, une analgésie inadéquate expose à l'hypoventilation alvéolaire et à l'inefficacité de la toux, à l'encombrement bronchique, aux atélectasies et, à terme, à la défaillance ventilatoire et à la surinfection éventuelle des lésions parenchymateuses. L'utilisation d'une analgésie multimodale par voie orale et/ou parentérale est donc indispensable. Une titration morphinique est recommandée en cas de douleur intense avec un objectif de soulagement adéquat $(E V A \leq 3)$ et rapide. En cas de besoin de mobilisation du patient et d'analgésie insuffisante malgré une titration morphinique bien conduite, les experts recommandent l'emploi de kétamine à doses analgésiques [6]. Lors de la réalisation de gestes invasifs (drainage pleural particulièrement), une anesthésie locale, voire une analgésie-sédation, peut s'avérer nécessaire. L'emploi d'une sédation impose de rester dans des conditions de sécurité indispensables chez ces patients à l'estomac plein et présentant classiquement un iléus réflexe. Le risque d'inhalation est majeur et peut avoir des conséquences dramatiques sur un poumon traumatisé.

Lorsque la douleur n'est pas contrôlée dans les 12 heures suivant le traumatisme, ou chez les patients à fort potentiel d'aggravation (pathologie pulmonaire préalable, lésions pariétales sévères...), il est indispensable de pouvoir proposer une solution d'anesthésie locorégionale [6]. L'expertise d'un anesthésiste-réanimateur est alors requise afin de pouvoir proposer une analgésie péridurale thoracique en cas de lésions complexes multiétagées et/ou bilatérales. En comparaison avec l'analgésie morphinique par PCA, l'analgésie péridurale a été montrée comme permettant une amélioration quotidienne de la force inspiratoire maximale et une augmentation de $45 \%$ du volume courant au cours des trois jours suivant sa mise en place. Ces mêmes paramètres déclinent sur la même période avec une baisse du volume courant de $56 \%$ lors d'une analgésie par PCA morphinique. Parallèlement, aux doses analgésiques employées, la stabilité hémodynamique n'est pas compromise [29]. En 2004, Bulger et al. ont par ailleurs montré que l'analgésie péridurale était associée à une incidence plus faible de survenue de pneumopathies au cours de l'évolution des traumatismes thoraciques, en comparaison avec l'analgésie intraveineuse par PCA morphinique (18 vs $38 \%$ ) [30].

La péridurale a été définie comme la technique d'analgésie optimale des traumatismes thoraciques fermés, avec atteinte pariétale supérieure à quatre côtes, chez les patients de plus de 65 ans avec des comorbidités. La conférence française d'experts de 2015 recommande également l'emploi du bloc paravertébral, avec mise en place d'un cathéter, au mieux sous contrôle échographique, lors de la présence de fractures de côtes unilatérales $[6,31,32]$. Le choix se portera sur la péridurale ou sur le bloc paravertébral selon l'existence de lésions associées, en particulier du système nerveux central et des troubles de l'hémostase. L'existence d'une pathologie cérébrale traumatique associée contre-indique l'abord périmédullaire, de même que la présence d'une pathologie de l'hémostase constitutionnelle ou acquise. Enfin, la stabilité hémodynamique et le blocage sympathique cardiaque induit par la péridurale thoracique doivent être pris en considération, même s'il n'est pas décrit d'augmentation des doses de catécholamines après péridurale thoracique [29].

D'autres techniques d'analgésie locorégionale peuvent être proposées en appoint de l'analgésie multimodale intraveineuse (bloc du serratus, bloc de la paroi thoracique, bloc sous-rhomboïdal...) en fonction du nombre, de la topographie et de l'étendue des lésions pariétales. Elles nécessitent une expertise approfondie en anesthésie locorégionale et une réalisation technique sous contrôle échographique.

\section{Détresse respiratoire et assistance ventilatoire}

Les traumatismes des muscles pariétaux, du diaphragme, des côtes, du sternum altèrent la mécanique ventilatoire normale, parfois même en générant des mouvements paradoxaux de la paroi thoracique (volets) qui conduisent systématiquement à la survenue d'une hypoventilation alvéolaire. Celle-ci est majorée par l'inhibition réflexe de l'inspiration profonde liée à la douleur et par l'absence de maintien de la vacuité pleurale nécessaire à une expansion pulmonaire correcte. Parallèlement à cette hypoventilation se développe une 
hypoxémie par shunt intrapulmonaire, secondaire à l'apparition d'atélectasies (collapsus pulmonaire passif lié à un épanchement, à l'hypoventilation, au drainage bronchique insuffisant), ou par lésion pulmonaire directe dans les cas de contusion pulmonaire avec destruction plus ou moins importante du parenchyme. L'inflammation pulmonaire et systémique peut majorer par ailleurs ce shunt intrapulmonaire, en inhibant la vasoconstriction pulmonaire hypoxique physiologique. Les prostaglandines, générées par la cyclooxygénase, ont été montrées comme pouvant inhiber cette vasoconstriction pulmonaire hypoxique et perturber la réponse normale adaptative qui tend à améliorer les rapports ventilation/perfusion en diminuant le shunt pulmonaire [33]. S'associent donc à des degrés divers, et plus ou moins rapidement après le traumatisme, l'effet shunt et l'hypoventilation alvéolaire, avec pour résultante une hypoxémie et une hypercapnie immédiate ou retardée par fatigue respiratoire.

\section{Ventilation}

La nécessité de recourir à l'assistance ventilatoire est, de ce fait, extrêmement fréquente dans les traumatismes graves et justifie une surveillance en soins continus ou en réanimation et la mise en œuvre de techniques d'assistance respiratoire adaptées, bien conduites et sans délai. Comme dans toute atteinte respiratoire, l'accent doit être mis sur les techniques non invasives, de manière à limiter les complications liées à l'intubation et à la ventilation invasive et la morbidité qui y sont associées [34,35].

Une fois obtenue une analgésie efficace, la première des prises en charge ventilatoires consiste en une kinésithérapie intense et pluriquotidienne, visant à une clairance optimale des sécrétions de l'arbre trachéobronchique. Le positionnement du patient en position assise (en fonction des lésions associées particulièrement rachidiennes qui seront mises en évidence par le bilan exhaustif), les techniques d'accélération du flux expiratoire, de toux assistée (cough assist) et la spirométrie incitative font partie de l'arsenal thérapeutique indispensable.

L'oxygénothérapie est indissociable de la prise en charge des patients hypoxémiques. Le développement récent de l'oxygénothérapie à haut débit dans les services de réanimation, pour la prise en charge des patients hypoxémiques, est vraisemblablement d'un intérêt majeur dans ces circonstances bien qu'il existe peu d'arguments scientifiques, à l'heure actuelle, dans le contexte particulier de la traumatologie thoracique [36-39]. Cependant, cette technique permet une humidification optimale des voies aériennes, une meilleure tolérance et un confort amélioré pour le patient, gage d'une efficacité et d'une observance adéquate pour une durée prolongée. Enfin, l'existence d'un effet Pep (pression expiratoire positive) modéré pour des débits supérieurs à $50 \mathrm{l} / \mathrm{min}$ est bénéfique pour la prise en charge. Cette technique peut être utilisée en association avec des séances itératives de ventilation non invasive (VNI) en ventilation spontanée, avec aide inspiratoire et pression positive télé-expiratoire (VSAI + Pep), afin de prévenir et/ou traiter une défaillance respiratoire avec survenue d'une hypoventilation alvéolaire et hypercapnie. En effet, la VNI a montré son bénéfice sur la mortalité et la morbidité des traumatisés du thorax. Chez ces patients hypoxémiques, la VNI permet de réduire le recours à l'intubation, diminue l'incidence de survenue des pneumopathies et diminue la durée de séjour hospitalier ainsi que la mortalité $[34,35,40-43]$. La conférence d'experts récente recommande qu'en milieu hospitalier, en présence d'une hypoxémie, après réalisation d'une tomodensitométrie et d'un drainage thoracique si nécessaire, soit réalisée une VNI dans un environnement disposant d'une surveillance continue [6]. L'absence d'amélioration clinique et gazométrique (hypoxémie et/ou aggravation de l'hypoventilation alvéolaire) dans l'heure qui suit l'instauration bien conduite de cette technique doit faire envisager le recours à la ventilation invasive sans retarder de manière délétère le moment de la décision [44].

Lorsque la ventilation invasive est inéluctable malgré l'analgésie optimale, le rétablissement de la vacuité pleurale (si nécessaire), la kinésithérapie et la VNI, le patient doit être intubé. Cette intubation doit répondre aux critères de l'intubation en urgence chez un patient à haut risque d'inhalation et doit donc être réalisée en séquence rapide. On veillera par ailleurs à prendre en compte les lésions associées éventuelles et à appliquer les techniques adaptées pour éviter toute complication de l'intubation, notamment en cas de traumatisme rachidien. La ventilation invasive instaurée après intubation orotrachéale doit répondre aux critères d'une ventilation protective $[45,46]$. En effet, le poumon contus est un poumon pathologique, fragile, pour lequel l'application des recommandations de ventilation protective, au même titre qu'un poumon de SDRA, limite les lésions induites par la ventilation mécanique [47]. L'inflammation, les lésions de stretch alvéolaire, les baro- et volotraumatismes doivent être évités [48]. Le volume courant doit être réglé à $6 \mathrm{ml} / \mathrm{kg}$ de poids idéal théorique, avec un objectif de pression de plateau inférieur à $30 \mathrm{cmH}_{2} \mathrm{O}$. Une attention particulière sera portée à la pression motrice. Elle correspond à la différence entre la Pep et la pression de plateau. Diminuer cette variable à pression de plateau égale pourrait avoir un effet sur la mortalité [49]. Dans ces circonstances traumatiques, la présence éventuelle de lésions abdominales associées peut justifier la réalisation d'une mesure de la pression abdominale (via la pression intra-vésicale), afin de déceler des conditions favorisant une altération de la course diaphragmatique. L'utilisation de la Pep sera titrée au moins égale à $5 \mathrm{cmH}_{2} \mathrm{O}$ et adaptée à l'oxygénation et à la tolérance hémodynamique. Le recrutement alvéolaire devra permettre la baisse de la $\mathrm{FiO}_{2}$ avec des objectifs de $\mathrm{SpO} 2$ entre 88 et $95 \%$ et de $\mathrm{PaO} 2$ entre 60 et 
90 mmHg (en dehors d'un traumatisme crânien associé), afin de limiter la toxicité tissulaire de l'hyperoxie. Cette adaptation de la Pep nécessite l'emploi d'un monitorage hémodynamique adapté pour évaluer le débit cardiaque du patient, la précharge ventriculaire, la survenue d'une insuffisance ventriculaire droite, la présence d'un foramen ovale perméable. Lors d'une ventilation invasive d'un traumatisé thoracique avec contusion pulmonaire sévère, la prise en charge ventilatoire emprunte les mêmes techniques, les mêmes précautions et les mêmes objectifs que la ventilation d'un patient présentant un SDRA. L'évolution pourra nécessiter des séances de décubitus ventral, l'adjonction de monoxyde d'azote, voire une assistance circulatoire par ECMO (extracorporeal membrane oxygenation). Chaque situation sera discutée en fonction des lésions traumatiques extrathoraciques associées éventuelles, lesquelles peuvent nécessiter des objectifs affinés ou représenter des contre-indications à certaines alternatives thérapeutiques (hypercapnie permissive et traumatisme crânien, décubitus ventral et traumatisme instable du rachis et/ou hypertension intracrânienne, ECMO et lésion à potentiel hémorragique...).

\section{Drainage pleural}

Le drainage pleural revêt une importance majeure en traumatologie thoracique. Il a pour rôle de rétablir la vacuité pleurale, restaurant le rôle physiologique de la plèvre qui est d'assurer la transmission à l'échangeur pulmonaire des mouvements générés par la paroi thoracique. L'expansion pulmonaire est directement influencée par cette vacuité pleurale. Le drainage pleural est un geste fréquent en réanimation, mais parfois mal maitrisé et grevé d'une morbidité lourde lorsque sa réalisation est incorrecte et/ou approximative. En effet, l'analyse tomodensitométrique de la pose de 106 drains thoraciques chez 63 patients a révélé $21 \%$ de drains intrascissuraux et $9 \%$ de drains intraparenchymateux, entraînant des saignements pulmonaires, des fistules bronchopleurales et des abcès pulmonaires [50]. Un des facteurs principaux associés à ces malpositions était la réalisation incorrecte du drainage, sans dissection au doigt, mais avec une insertion « au trocart », à l'aveugle. De grands principes simples méritent d'être rappelés. On ne doit jamais introduire un drain en dessous du niveau du mamelon, ni en position plus interne que le mamelon, ni par un orifice de drainage préalable, ni par l'orifice d'une plaie. Si on trace une ligne verticale passant par le milieu de la clavicule et le mamelon et une ligne horizontale passant par le mamelon, on définit quatre quadrants sur le thorax antérieur du patient. Seul le quadrant supéro-externe peut être le siège du point d'entrée d'un drainage thoracique [51]. Le drainage par voie antérieure doit être réalisé au deuxième espace intercostal, sur la ligne médioclaviculaire. Le risque, s'il est trop interne, est de léser l'artère mammaire interne qui chemine deux cen- timètres en dehors du bord latéral du sternum. L'échographie pleuropulmonaire prend tout son intérêt dans la sécurisation du geste de drainage pleural, en vérifiant le site d'insertion choisi et en sécurisant la pose [52]. Enfin, il n'y a pas d'indication à une antibioprophylaxie lors de la pose d'un drainage thoracique dans un traumatisme thoracique fermé [6].

On choisira les drains les moins agressifs pour le poumon. La technique de pose doit faire appel à une technique stricte avec dissection des plans sous-cutanés à la pince, puis ouverture de la plèvre pariétale « au doigt ». Cela garantit l'absence de lésion du poumon sous-jacent. L'insertion du drain se fait ensuite en évitant de faire pénétrer le mandrin au-delà de la plèvre pariétale. Les drains souples perforés en silicone, introduits par un trocart de Monod, sont les moins pourvoyeurs de lésions pulmonaires. Les drains à trocart interne, de type "drain de Jolly », exposent en revanche, par leur mandrin inclus dans le drain, au risque de lésion endothoracique, si celui-ci n'est pas immobilisé dès le passage de la plèvre pariétale. Les drains en silicone sont plus souples, moins douloureux et moins aptes à cailloter. Enfin, les drains de petite taille, introduits au travers d'une aiguille de gros calibre à bout acéré, doivent être proscrits, car trop dangereux pour le parenchyme pulmonaire [51].

Dans le contexte traumatique, tout épanchement aérique et/ou hématique significatif justifie un drainage pleural. Les épanchements aériques sont fréquemment associés à un bullage persistant pendant plusieurs jours et associés à un certain degré d'hémothorax, ce qui impose le drainage et exclut l'exsufflation simple. En cas de pneumothorax isolé, on utilisera des drains de calibre charrière 18 à 24 . Dans les situations mettant en évidence un hémothorax, l'usage est d'utiliser des drains de gros calibre (charrière 28 à 32) afin d'éviter qu'ils ne se bouchent avec des caillots et drainent incomplètement la plèvre [53]. La qualité du drainage initial est un facteur important dans la prévention de la survenue ultérieure de complications pulmonaires. Un hémothorax résiduel est corrélé à une plus forte proportion de complications précoces à type d'empyème pleural [54] et tardives sous forme de fibrose et d'atélectasies. Les hémothorax retardés, survenant fréquemment à $\mathrm{j} 3$ de fractures de côtes multiples (plus de trois le plus souvent), peuvent être drainés avec des drains de petite taille [55]. Des drains de type drain de Fuhrman permettent une vacuité pleurale correcte, du fait du caractère très fluide de ce type d'épanchement. Il s'agit, en effet, d'un suintement lent des différents foyers fracturaires, avec une fibrinolyse pleurale et une absence de caillotage contrairement aux hémothorax massifs immédiats.

Une attention particulière doit être accordée à la décompression d'un pneumothorax compressif en cas de détresse respiratoire ou hémodynamique avec forte suspicion de tamponnade gazeuse [6]. En effet, la survenue d'un événement de ce type ne justifie pas le drainage pleural en urgence. L'urgence est à la décompression de la plèvre. Il faut 
éliminer la surpression intrapleurale, en ramenant la pression à la pression atmosphérique afin de lever la tamponnade, puis réaliser le drainage rapidement, mais au calme, après stabilisation hémodynamique et respiratoire, et rétablir la dépression de la plèvre par aspiration. L'exsufflation est un geste de sauvetage, mais sa technique ne doit pas être aléatoire. Elle doit être réalisée au deuxième espace intercostal, sur la ligne médioclaviculaire, ou mieux, si le creux axillaire est accessible et le bras mobilisable, au troisième ou au quatrième espace, sur la ligne axillaire antérieure (moins de risque d'échec) [56]. En cas d'échec de l'exsufflation ou d'arrêt cardiorespiratoire, une thoracostomie axillaire est indiquée [57-59].

Enfin, le drainage pleural permet d'alerter sur une lésion potentiellement chirurgicale lors d'un bullage permanent du drain en aspiration avec fistule bronchopleurale ou lors du drainage d'un hémothorax révélant :

- en cas d'instabilité hémodynamique, un saignement actif dans le drain thoracique sans autre site de saignement ;

- en cas de stabilité hémodynamique, mais avec un débit sur le drain thoracique comme suit [6] :

- un volume à la pose de plus de $1500 \mathrm{ml}$ de sang d'emblée et plus de $200 \mathrm{ml}$ la première heure suivant la pose du drain,

- un volume à la pose de moins de $1500 \mathrm{ml}$ de sang d'emblée, mais un saignement persistant de plus de $200 \mathrm{ml} / \mathrm{h}$ pendant plus de trois heures.

Il convient alors de contacter une équipe de chirurgie cardiothoracique pour envisager une thoracotomie exploratrice d'hémostase.

\section{Fixation chirurgicale d'un volet costal}

Même lors de la prise en charge d'un patient victime d'un traumatisme fermé du thorax, il faut savoir discuter d'indications chirurgicales secondaires éventuelles avec une équipe chirurgicale, particulièrement pour la prise en charge sur le plan ventilatoire. Lorsque le patient a nécessité une ventilation invasive en raison de son atteinte thoracique ou de lésions associées, la présence d'un volet costal ou costosternal très mobile peut entraver le sevrage de la ventilation mécanique, en rendant inefficaces les efforts inspiratoires du patient. La fixation chirurgicale doit être envisagée lorsque le sevrage de la ventilation mécanique ne peut pas être réalisé dans les 36 heures qui suivent la résolution de l'événement ayant conduit à la nécessité de ventilation [6] (contrôle d'un état de choc hémorragique, réalisation d'interventions chirurgicales pour d'autres lésions abdominales, orthopédiques, amélioration parenchymateuse et gazométrique d'une contusion pulmonaire, etc.).

\section{Défaillance hémodynamique}

La défaillance circulatoire est fréquemment associée aux traumatismes thoraciques. Sa présence est plus habituelle dans les traumatismes majeurs avec indication d'exploration chirurgicale que dans les traumatismes « non chirurgicaux ». Les lésions les plus fréquentes sont les hémothorax massifs liés aux fractures de côtes multiples, les plaies pulmonaires hémorragiques, les tamponnades péricardiques et les lésions des gros vaisseaux. Il ne faut cependant pas négliger les situations dans lesquelles le patient initialement stable et semblant non chirurgical va nécessiter finalement une prise en charge chirurgicale : hémothorax qui ne se tarit pas après drainage, hémothorax d'apparition secondaire dans un contexte de rupture traumatique aortique...

En dehors de ces situations de traumatismes thoraciques chirurgicaux, une instabilité peut être liée à la présence d'une défaillance cardiaque par contusion myocardique avec une atteinte ventriculaire droite majoritairement, mais aussi ventriculaire gauche, dans les traumatismes par impact sternal direct ou par décélération violente. Les contusions myocardiques sont décelées par un faisceau d'arguments à la prise en charge : ascension de troponine I, troubles du rythme ou de la conduction à l'ECG, fuite valvulaire par rupture de pilier valvulaire ou dysfonction systolique à l'échographie. D'authentiques lésions coronaires peuvent, par ailleurs, être mises en évidence (dissection artérielle coronaire, rupture de plaque préexistante) [60]. Il existe alors une mise en circulation de troponine I significative et prolongée. Ces lésions (relativement rares) peuvent indiquer un geste thérapeutique endovasculaire ou de chirurgie cardiaque, dont le timing de la réalisation devra être discuté en prenant en considération les lésions associées éventuelles et leurs traitements spécifiques.

Très souvent, l'instabilité hémodynamique est liée à une lésion associée, pour laquelle la prise en charge thérapeutique (remplissage, catécholamines, transfusions) va nécessiter de prendre en compte l'atteinte pulmonaire.

Comme pour la ventilation, l'analogie entre la contusion pulmonaire, le poumon agressé et le SDRA est grande. Tout remplissage vasculaire non nécessaire à l'optimisation hémodynamique peut se révéler délétère pour les échanges pulmonaires. En 2006, une étude de l'ARDS Network a démontré un bénéfice en termes de durée de ventilation lorsque, au-delà des 48 premières heures, le remplissage vasculaire était géré de manière « conservatrice », raisonnée et argumentée versus une gestion « libérale », non monitorée et moins restrictive chez les patients présentant un SDRA. La probabilité de survie et la proportion de patients sevrés de la ventilation étaient significativement meilleures lorsque le remplissage vasculaire était optimisé [61]. De manière analogue, l'optimisation de la volémie chez les traumatisés du thorax nécessite un monitorage invasif par cathéter artériel, 
mesure invasive du débit cardiaque, évaluation échographique de la fonction cardiaque et des pressions de remplissage cardiaque. En fonction des associations lésionnelles extrathoraciques, une hémorragie abdominale ou pelvienne lors d'une fracture de la rate ou du bassin, ou la présence d'une sympatholyse cardiaque et/ou vasculaire lors d'un traumatisme médullaire nécessiteront des actions thérapeutiques ciblées différentes, avec des retentissements à évaluer systématiquement sur la surcharge pulmonaire induite.

\section{Conclusion}

Les traumatismes thoraciques revêtent des tableaux extrêmement variés en termes de gravité, de défaillances vitales générées, de type de lésion et d'association entre elles. Le point commun est le fort potentiel de morbidité justifiant une attitude toujours attentive et prudente. Le bilan lésionnel doit être approfondi et rigoureux, afin d'éliminer les indications dans lesquelles l'exploration chirurgicale est indispensable et ne doit pas être méconnue. La prise en charge n'en est pas moins cruciale lorsque le traumatisme est non chirurgical. L'analgésie correcte et rapide, la réalisation en toute sécurité des gestes invasifs, l'assistance ventilatoire adaptée et la gestion optimale de l'hémodynamique doivent permettre de minimiser la morbidité et l'aggravation secondaire fréquente. Enfin, la précocité et l'intensité de la prise en charge doivent limiter au maximum les séquelles respiratoires à long terme, en réduisant, autant que possible, l'amputation du parenchyme pulmonaire et les déformations thoraciques. Il ne faut jamais perdre de vue que ces patients sont le plus souvent des polytraumatisés, et, à ce titre, le bilan de toutes les lésions potentiellement associées est indispensable. La non-prise en compte de ces lésions associées peut être responsable de conséquences mortelles ou à fort potentiel de morbidité par leur présence propre ou par les implications qu'elles génèrent dans la réanimation à visée pulmonaire.

Liens d'intérêts : Les auteurs déclarent ne pas avoir de liens d'intérêts.

\section{Références}

1. Karmy-Jones R, Jurkovich GJ, (2004) Blunt chest trauma. Curr Probl Surg 41: 211-380

2. Sauaia A, Moore FA, Moore EE, Moser KS, Brennan R, Read RA, Pons PT, (1995) Epidemiology of trauma deaths: a reassessment. J Trauma 38: 185-193

3. Avaro JP, Bonnet PM, (2011) The management of blunt chest trauma. Rev Mal Respir 28: 152-163

4. Wanek S, Mayberry JC, (2004) Blunt thoracic trauma: flail chest, pulmonary contusion, and blast injury. Crit Care Clin 20: 71-81
5. Jonsson A, Arvebo E, Schantz B, (1988) Intrathoracic pressure variations in an anthropomorphic dummy exposed to air blast, blunt impact, and missiles. J Trauma 28: S125-S131

6. SFAR/SFMU Cde, (2015) Traumatisme thoracique : prise en charge des 48 premières heures. Anesth Reanim 1: 272-287

7. Sartorius D, Le Manach Y, David JS, Rancurel E, Smail N, Thicoipe M, Wiel E, Ricard-Hibon A, Berthier F, Gueugniaud PY, Riou B, (2010) Mechanism, glasgow coma scale, age, and arterial pressure (MGAP): a new simple prehospital triage score to predict mortality in trauma patients. Crit Care Med 38: 831-837

8. Riou B, Thicoipe M, Atain-Kouadio P, (2002) Comment évaluer la gravité ? SAMU de France. Actualités en réanimation préhospitalière : le traumatisé grave. Paris, SFEM éditions. Vittel 115-28

9. Battle CE, Hutchings H, James K, Evans PA, (2013) The risk factors for the development of complications during the recovery phase following blunt chest wall trauma: a retrospective study. Injury 44: 1171-1176

10. Michelet $\mathrm{C},(2010)$ Traumatismes thoraciques $52^{\mathrm{e}}$ congrès national d'anesthésie et de réanimation Conférences d'essentiels, Paris

11. Raghavendran K, Davidson BA, Woytash JA, Helinski JD, Marschke CJ, Manderscheid PA, Notter RH, Knight PR, (2005) The evolution of isolated bilateral lung contusion from blunt chest trauma in rats: cellular and cytokine responses. Shock 24 : $132-138$

12. Rivkind AI, Siegel JH, Guadalupi P, Littleton M, (1989) Sequential patterns of eicosanoid, platelet, and neutrophil interactions in the evolution of the fulminant post-traumatic adult respiratory distress syndrome. Ann Surg 210: 355-372; discussion 372-373

13. Melton SM, Davis KA, Moomey CB Jr, Fabian TC, Proctor KG, (1999) Mediator-dependent secondary injury after unilateral blunt thoracic trauma. Shock 11: 396-402

14. Davis KA, Fabian TC, Croce MA, Proctor KG, (1999) Prostanoids: early mediators in the secondary injury that develops after unilateral pulmonary contusion. J Trauma 46: 824-831; discussion $831-832$

15. Miller PR, Croce MA, Bee TK, Qaisi WG, Smith CP, Collins GL, Fabian TC, (2001) ARDS after pulmonary contusion: accurate measurement of contusion volume identifies high-risk patients. J Trauma 51: 223-228; discussion 229-230

16. MacKenzie EJ, Rivara FP, Jurkovich GJ, Nathens AB, Frey KP, Egleston BL, Salkever DS, Scharfstein DO, (2006) A national evaluation of the effect of trauma-center care on mortality. $\mathrm{N}$ Engl J Med 354: 366-378

17. Yeguiayan JM, Garrigue D, Binquet C, Jacquot C, Duranteau J, Martin C, Rayeh F, Riou B, Bonithon-Kopp C, Freysz M, (2011) Medical pre-hospital management reduces mortality in severe blunt trauma: a prospective epidemiological study. Crit Care 15: R34

18. Harrois A, Hamada S, Laplace C, Duranteau J, Vigue B, (2013) The initial management of severe trauma patients at hospital admission. Ann Fr Anesth Reanim 32: 483-491

19. Rocco M, Carbone I, Morelli A, Bertoletti L, Rossi S, Vitale M, Montini L, Passariello R, Pietropaoli P, (2008) Diagnostic accuracy of bedside ultrasonography in the ICU: feasibility of detecting pulmonary effusion and lung contusion in patients on respiratory support after severe blunt thoracic trauma. Acta Anaesthesiol Scand 52: 776-784

20. Brooks A, Davies B, Smethhurst M, Connolly J, (2004) Emergency ultrasound in the acute assessment of haemothorax. Emerg Med J 21: 44-46

21. Peytel E, Menegaux F, Cluzel P, Langeron O, Coriat P, Riou B, (2001) Initial imaging assessment of severe blunt trauma. Intensive Care Med 27: 1756-1761

22. Zhang M, Liu ZH, Yang JX, Gan JX, Xu SW, You XD, Jiang GY, (2006) Rapid detection of pneumothorax by ultrasonography in patients with multiple trauma. Crit Care 10: R112 
23. Deunk J, Brink M, Dekker HM, Kool DR, Blickman JG, van Vugt AB, Edwards MJ, (2009) Routine versus selective multidetector-row computed tomography (MDCT) in blunt trauma patients: level of agreement on the influence of additional findings on management. J Trauma 67: 1080-1086

24. Scaglione M, Pinto A, Pedrosa I, Sparano A, Romano L, (2008) Multi-detector row computed tomography and blunt chest trauma. Eur J Radiol 65: 377-388

25. Huber-Wagner S, Lefering R, Qvick LM, Korner M, Kay MV, Pfeifer KJ, Reiser M, Mutschler W, Kanz KG, (2009) Effect of whole-body CT during trauma resuscitation on survival: a retrospective, multicentre study. Lancet 373: 1455-1461

26. Hamada SR, Delhaye N, Kerever S, Harrois A, Duranteau J, (2016) Integrating eFAST in the initial management of stable trauma patients: the end of plain film radiography. Ann Intensive Care 6: 62

27. Vignon P, Martaille JF, Francois B, Rambaud G, Gastinne H, (2005) Transesophageal echocardiography and therapeutic management of patients sustaining blunt aortic injuries. J Trauma 58: $1150-1158$

28. Goarin JP, Cluzel P, Gosgnach M, Lamine K, Coriat P, Riou B, (2000) Evaluation of transesophageal echocardiography for diagnosis of traumatic aortic injury. Anesthesiology 93: 1373-1377

29. Moon MR, Luchette FA, Gibson SW, Crews J, Sudarshan G, Hurst JM, Davis K, Jr., Johannigman JA, Frame SB, Fischer JE, (1999) Prospective, randomized comparison of epidural versus parenteral opioid analgesia in thoracic trauma. Ann Surg 229: 684-691

30. Bulger EM, Edwards T, Klotz P, Jurkovich GJ, (2004) Epidural analgesia improves outcome after multiple rib fractures. Surgery 136: $426-430$

31. Ding X, Jin S, Niu X, Ren H, Fu S, Li Q, A comparison of the analgesia efficacy and side effects of paravertebral compared with epidural blockade for thoracotomy: an updated meta-analysis. PLoS One 9: e96233

32. Karmakar MK, (2001) Thoracic paravertebral block. Anesthesiology $95: 771-780$

33. Leeman M, Delcroix M, Vachiery JL, Melot C, Naeije R, (1992) Blunted hypoxic vasoconstriction in oleic acid lung injury: effect of cyclooxygenase inhibitors. J Appl Physiol (1985) 72: 251-258

34. Hernandez G, Fernandez R, Lopez-Reina P, Cuena R, Pedrosa A, Ortiz R, Hiradier P, (2010) Noninvasive ventilation reduces intubation in chest trauma-related hypoxemia: a randomized clinical trial. Chest 137: 74-80

35. Duggal A, Perez P, Golan E, Tremblay L, Sinuff T, (2013) Safety and efficacy of noninvasive ventilation in patients with blunt chest trauma: a systematic review. Crit Care 17: R142

36. Mundel T, Feng S, Tatkov S, Schneider H, (1985) Mechanisms of nasal high flow on ventilation during wakefulness and sleep. $\mathrm{J}$ Appl Physiol 114: 1058-1065

37. Braunlich J, Beyer D, Mai D, Hammerschmidt S, Seyfarth HJ, Wirtz H, (2013) Effects of nasal high flow on ventilation in volunteers, COPD and idiopathic pulmonary fibrosis patients. Respiration 85: 319-325

38. Frat JP, Thille AW, Mercat A, Girault C, Ragot S, Perbet S, Prat G, Boulain T, Morawiec E, Cottereau A, Devaquet J, Nseir S, Razazi K, Mira JP, Argaud L, Chakarian JC, Ricard JD, Wittebole X, Chevalier S, Herbland A, Fartoukh M, Constantin JM, Tonnelier JM, Pierrot M, Mathonnet A, Beduneau G, DeletageMetreau C, Richard JC, Brochard L, Robert R, (2015) High-flow oxygen through nasal cannula in acute hypoxemic respiratory failure. N Engl J Med 372: 2185-2196

39. Stephan F, Barrucand B, Petit P, Rezaiguia-Delclaux S, Medard A, Delannoy B, Cosserant B, Flicoteaux G, Imbert A, Pilorge C, Berard L, (2015) High-flow nasal oxygen vs noninvasive positive airway pressure in hypoxemic patients after cardiothoracic surgery: a randomized clinical trial. JAMA 313: 2331-2339

40. Antonelli M, Conti G, Rocco M, Bufi M, De Blasi RA, Vivino G, Gasparetto A, Meduri GU, (1998) A comparison of noninvasive positive-pressure ventilation and conventional mechanical ventilation in patients with acute respiratory failure. $\mathrm{N}$ Engl $\mathrm{J}$ Med 339: 429-435

41. Bolliger CT, Van Eeden SF, (1990) Treatment of multiple rib fractures. Randomized controlled trial comparing ventilatory with nonventilatory management. Chest 97: 943-948

42. Gunduz M, Unlugenc $\mathrm{H}$, Ozalevli $\mathrm{M}$, Inanoglu $\mathrm{K}$, Akman $\mathrm{H}$, (2005) A comparative study of continuous positive airway pressure (CPAP) and intermittent positive pressure ventilation (IPPV) in patients with flail chest. Emerg Med J 22: 325-329

43. Ferrer M, Esquinas A, Leon M, Gonzalez G, Alarcon A, Torres A, (2003) Noninvasive ventilation in severe hypoxemic respiratory failure: a randomized clinical trial. Am J Respir Crit Care Med 168: 1438-1444

44. Truwit JD, Bernard GR, (2004) Noninvasive ventilation--don't push too hard. N Engl J Med 350: 2512-2515

45. Amato MB, Barbas CS, Medeiros DM, Magaldi RB, Schettino GP, Lorenzi-Filho G, Kairalla RA, Deheinzelin D, Munoz C, Oliveira R, Takagaki TY, Carvalho CR, (1998) Effect of a protective-ventilation strategy on mortality in the acute respiratory distress syndrome. N Engl J Med 338: 347-354

46. 1999) International consensus conferences in intensive care medicine: Ventilator-associated Lung Injury in ARDS. This official conference report was cosponsored by the American Thoracic Society, the European Society of Intensive Care Medicine, and the Société de réanimation de langue française, and was approved by the ATS Board of Directors, July 1999. Am J Respir Crit Care Med 160: 2118-2124

47. Schultz MJ, (2008) Lung-protective mechanical ventilation with lower tidal volumes in patients not suffering from acute lung injury: a review of clinical studies. Med Sci Monit 14: RA22-RA26

48. Dos Santos CC, Slutsky AS, (2000) Invited review: mechanisms of ventilator-induced lung injury: a perspective. J Appl Physiol (1985) 89: 1645-1655

49. Amato MB, Meade MO, Slutsky AS, Brochard L, Costa EL, Schoenfeld DA, Stewart TE, Briel M, Talmor D, Mercat A, Richard JC, Carvalho CR, Brower RG, (2015) Driving pressure and survival in the acute respiratory distress syndrome. N Engl J Med 372: 747-755

50. Remerand F, Luce V, Badachi Y, Lu Q, Bouhemad B, Rouby JJ, (2007) Incidence of chest tube malposition in the critically ill: a prospective computed tomography study. Anesthesiology 106: $1112-1119$

51. Tazarourte K, Laplace C, Atchabahian A, (2011) Chest tubes. In: Tubaro M, Danchin M, Filippatos G, et al The ESC textbook of intensive and acute cardiac care. Oxford University Press, New York, pp 258-265

52. Havelock T, Teoh R, Laws D, Gleeson F, (2010) Pleural procedures and thoracic ultrasound: British Thoracic Society Pleural Disease Guideline. Thorax 65: ii61-ii76

53. Contou D, Razazi K, Katsahian S, Maitre B, Mekontso-Dessap A, Brun-Buisson C, Thille AW, (2012) Small-bore catheter versus chest tube drainage for pneumothorax. Am J Emerg Med 30: $1407-1413$

54. Karmy-Jones R, Holevar M, Sullivan RJ, Fleisig A, Jurkovich GJ, (2008) Residual hemothorax after chest tube placement correlates with increased risk of empyema following traumatic injury. Can Respir J 15: 255-258

55. Tsai WK, Chen W, Lee JC, Cheng WE, Chen CH, Hsu WH, Shih $\mathrm{CM}$, (2006) Pigtail catheters vs large-bore chest tubes for management of secondary spontaneous pneumothoraces in adults. Am J Emerg Med 24: 795-800 
56. Laan DV, Vu TD, Thiels CA, Pandian TK, Schiller HJ, Murad MH, Aho JM, (2016) Chest wall thickness and decompression failure: A systematic review and meta-analysis comparing anatomic locations in needle thoracostomy. Injury 47 : 797-804

57. Aylwin CJ, Brohi K, Davies GD, Walsh MS, (2008) Pre-hospital and in-hospital thoracostomy: indications and complications. Ann R Coll Surg Engl 90: 54-57

58. Barton ED, Epperson M, Hoyt DB, Fortlage D, Rosen P, (1995) Prehospital needle aspiration and tube thoracostomy in trauma victims: a six-year experience with aeromedical crews. J Emerg Med 13: 155-163
59. Massarutti D, Trillo G, Berlot G, Tomasini A, Bacer B, D’Orlando L, Viviani M, Rinaldi A, Babuin A, Burato L, Carchietti E, (2006) Simple thoracostomy in prehospital trauma management is safe and effective: a 2-year experience by helicopter emergency medical crews. Eur J Emerg Med 13: 276-280

60. Edouard AR, Felten ML, Hebert JL, Cosson C, Martin L, Benhamou D, (2004) Incidence and significance of cardiac troponin I release in severe trauma patients. Anesthesiology 101: 1262-1268

61. Wiedemann HP, Wheeler AP, Bernard GR, Thompson BT, Hayden D, deBoisblanc B, Connors AFJr, Hite RD, Harabin AL, (2006) Comparison of two fluid-management strategies in acute lung injury. N Engl J Med 354: 2564-2575 